\title{
Amostragem, diversidade e sazonalidade de Hemerobiidae (Neuroptera) em Coffea arabica L. cv. Obatã (Rubiaceae)
}

\author{
Rogéria Inês Rosa Lara ${ }^{1}$, Sérgio de Freitas², Nelson Wanderley Periotoํㄹ \& Claudia Cristina Paro de Paz ${ }^{1}$
}

1APTA Ribeirão Preto, Rua Peru 1472-A, 14075-310, Ribeirão Preto-SP, Brasil. rirlara@aptaregional.sp.gov.br; rirlara@yahoo.com.br ${ }^{2}$ Departamento de Fitossanidade, Faculdade de Ciências Agrárias e Veterinárias, UNESP, Via de Acesso Prof. Paulo Donato Castellane, s/n, 14884-900, Jaboticabal-SP, Brasil. serfre@fcav.unesp.br

\begin{abstract}
Sampling, diversity and seasonal occurrence of Hemerobiidae (Neuroptera) in Coffea arabica L. cv. Obatã (Rubiaceae). This study evaluated sampling methods, seasonality and diversity of the hemerobiids associated to Coffea arabica L. cv. Obatã over a one-year period in Cravinhos, São Paulo, Brazil. The collecting methods were: sweeping net, light trap and Möericke trap. 489 hemerobiids belonging to four genera, were collected: Nusalala (231 individuals / $47.2 \%$ of the hemerobiids collected), Megalomus (110/22.5\%), Hemerobius (104/21.3\%) and Sympherobius (44 / 9\%). The sweeping net seems to be the most efficient method of sampling to capture Hemerobiidae and the Möericke trap presented the higher value of diversity $\left(H^{\prime}=0,56\right)$ and equitability $(\mathrm{J}=0,93)$. The hemerobiids were recorded in the area along the entire year. The highest abundance occurred from August to March (end of winter, spring and summer) and the population peak was in January (mid-summer). Megalomus presented positive and significant correlations $(\mathrm{p}<0.05)$ with the rainfall and maximum and minimum temperatures; Nusalala with the maximum and minimum temperatures and Sympherobius only with the maximum temperature.
\end{abstract}

KEYWORDS. Hemerobius; Megalomus; Nusalala; predator; Sympherobius.

\begin{abstract}
RESUMO. Amostragem, diversidade e sazonalidade de Hemerobiidae (Neuroptera) em Coffea arabica L. cv. Obatã (Rubiaceae). Este estudo teve por objetivo avaliar métodos de amostragem, abundância sazonal e diversidade da população de Hemerobiidae associada a cultivo de café Coffea arabica L. cv. Obatã em Cravinhos, São Paulo, Brasil. Para tanto foram realizadas amostragens semanais no período de maio de 2005 a abril de 2006. Os métodos de amostragem utilizados foram: rede de varredura e armadilhas de Möericke e luminosa. Foram coletados 491 exemplares de Hemerobiidae pertencentes a quatro gêneros: Nusalala (231 espécimes / 47,2\% do total de hemerobiídeos coletados), Megalomus (110 / 22,5\%), Hemerobius (104/21,3\%) e Sympherobius (44/9\%). A rede de varredura foi a mais eficiente para a captura de Hemerobiidae e a armadilha de Möericke foi o método de amostragem que apresentou os maiores valores de diversidade $\left(H^{\prime}=0,56\right)$ e de equitabilidade $(\mathrm{J}=0,93)$. Os hemerobiídeos estiveram presentes na área estudada durante o ano todo; as maiores freqüências foram registradas entre agosto e março (final do inverno, primavera e verão) e o maior pico populacional ocorreu em janeiro (na metade do verão). Megalomus apresentou correlação positiva e significativa ( $\mathrm{p}<$ 0,05) com a precipitação pluviométrica e as temperaturas máxima e mínima; Nusalala com as temperaturas máxima e mínima e, Sympherobius apenas com a temperatura máxima.
\end{abstract}

PALAVRAS-CHAVE. Hemerobius; Megalomus; Nusalala; predador; Sympherobius.

No agroecossistema cafeeiro ocorrem diversas espécies de artrópodes que causam prejuízos aos cafeeiros. Como pragachave destaca-se o bicho-mineiro Leucoptera coffeella (Guérin-Mèneville, 1842) (Lepidoptera, Lyonetiidae) e, como pragas secundárias, ácaros e cochonilhas (Reis et al. 2002). O bicho-mineiro é um dos principais problemas fitossanitários da cafeicultura brasileira (Reis et al. 2002) e seus danos podem limitar economicamente tal atividade (Gravena 1983; Fragoso et al. 2001). Estudos realizados por Parra et al. (1977), Gravena (1983) e Reis et al. (2002) indicaram as vespas como inimigos naturais-chave de L. coffeella. A atividade predatória do bichomineiro por outros agentes foi abordada por Ecole et al. (2002) que avaliaram a predação de $L$. coffeella por Chrysoperla externa (Hagen, 1861) (Neuroptera, Chrysopidae), grupo taxonômico afim de Hemerobiidae (Neuroptera).

Os Hemerobiidae, tanto larvas quanto adultos, alimentamse de ampla variedade de pequenos artrópodes sugadores, principalmente de afídeos, coccídeos, psilídeos, ácaros e outras espécies de corpo macio (Carpenter 1940; Monserrat 1990). Estudos sobre hemerobiídeos presentes em cultivos agrícolas são, em sua maioria, provenientes de países europeus e da América do Norte; são poucas as informações para a região Neotropical.

Szentkirályi $(1989,1992)$ estudou a dinâmica populacional e a diversidade de espécies de hemerobiídeos em agroecossistemas e florestas na Hungria e, em 1989, relatou a ocorrência de 12 espécies de hemerobiídeos, quatro delas dominantes: Micromus angulatus (Stephens, 1836), M. variegatus (Fabricius, 1793), Hemerobius humulinus Linnaeus, 1758 e Wesmaelius subnebulosus (Stephens, 1836), associadas a cultivos de milho. Em 1992, Szentkirályi observou que $H$. humulinus, M. angulatus, M. variegatus, Psectra diptera (Burmeister, 1839) e Sympherobius pygmaeus (Rambur, 1842) foram as espécies de hemerobiídeos mais abundantes em pomares de maçãs e em florestas e que seu pico populacional ocorreu em julho e agosto (verão no hemisfério norte). 
Na França, segundo Trouvé et al. (2002), M. variegatus, M. angulatus, $H$. humulinus e H. lutescens Fabricius, 1793 foram as espécies mais abundantes em cultivos de batata, feijão, maçã e morango, com pico populacional também no verão do hemisfério norte.

No Brasil, o conhecimento da fauna de hemerobiídeos é limitado e as informações a respeito deste grupo de insetos são provenientes de estudos taxonômicos (Penny \& Monserrat 1983; Souza 1999; Lara \& Freitas 2002, 2003), de registros de ocorrência (Chagas et al. 1982; Lara \& Perioto 2003) e de estudos de biologia (Souza et al. 1989; Souza et al. 1990; Souza \& Ciociola 1995, 1997).

A utilização de diferentes métodos de coleta tem um importante papel na amostragem de populações de insetos em campo (Szentkirályi 2002) e, para a captura de hemerobiídeos, são relatadas diferentes técnicas de coletas. $\mathrm{O}$ uso da rede de varredura da vegetação é indicado para a captura de espécies da maior parte das ordens associadas à vegetação, o que inclui espécies de insetos predadores de fitófagos, como os neurópteros (Almeida et al. 1998). Lara \& Freitas (2002) e Lara $\&$ Perioto (2003) capturaram grande número de exemplares de hemerobiídeos em cultivos de algodão, café e soja através de armadilhas de Möericke; não se conhece a razão pela qual estes predadores são atraídos por este tipo de armadilha. Trouvé et al. (2002) e Ábrahám et al. (2003) realizaram estudos com diferentes métodos de amostragem. Os primeiros obtiveram melhores resultados na captura de hemerobiídeos com o uso de armadilha de vácuo e de rede de varredura enquanto que os segundos observaram que a captura daqueles insetos foi melhor com a utilização de armadilha de sucção, seguida pelas armadilhas luminosas (mod. Jermy) e de Malaise. Diversos estudos realizados na Hungria relataram a eficiência da armadilha luminosa (mod. Jermy) para o monitoramento de neurópteros (Szabó \& Szentkirályi 1981; Szentkirályi 1992, 1997). A utilização de armadilhas luminosas para a captura de hemerobiídeos deve-se ao hábito crepuscular deste grupo de insetos que apresentam fototropismo positivo.

O objetivo deste estudo foi avaliar três métodos de amostragem para a captura de hemerobiídeos, a abundância sazonal e a diversidade destes predadores associados a $C$. arabica cv. Obatã no município de Cravinhos, São Paulo, Brasil.

\section{MATERIALE MÉTODOS}

O experimento foi realizado em lavoura de café arábica cv. Obatã, de quatro anos de idade, plantada no espaçamento $4 \mathrm{X}$ $1 \mathrm{~m}$ na Fazenda Palmares $\left(21^{\circ} 18^{\prime} 54^{\prime \prime} \mathrm{S} / 47^{\circ} 47^{\prime} 39^{\prime \prime O}\right)$, no município de Cravinhos (SP).

Os adultos de hemerobiídeos foram amostrados semanalmente no período de maio/2005 a abril/2006 através de coletas passivas com armadilhas luminosas (mod. Jermy) e de Möericke. As coletas ativas, com rede de varredura, iniciaramse em agosto/2005 e se estenderam até abril/2006.

Neste estudo foram utilizadas duas armadilhas luminosas (mod. Jermy) construídas conforme descrição de Szentkirályi (2002), equipadas com lâmpadas incandescentes de 100 W controladas por fotocélulas. As armadilhas, distantes entre si por $50 \mathrm{~m}$, foram fixadas através de travessas metálicas a postes de energia elétrica existentes no interior da cultura, de forma que sua cobertura circular ficasse na altura do dossel das plantas e permaneceram ativas por dois períodos consecutivos do anoitecer até o amanhecer do dia seguinte.

Como armadilhas de Möericke foram utilizados pratos plásticos descartáveis, de coloração amarela, com $15 \mathrm{~cm}$ de diâmetro e 4,5 cm de altura; cerca de $2 / 3$ de seu volume foi preenchido por solução conservante (solução aquosa de formalina e detergente neutro a 1\%). As armadilhas de Möericke foram fixadas em estacas de madeira com auxílio de aros de arame conforme proposto por Perioto et al. (2000), de forma que suas bordas ficassem próximas à altura dos terços inferior e médio da planta. A distribuição das armadilhas na área experimental seguiu a metodologia indicada por Gravena (1992) para a avaliação de pragas e inimigos naturais do cafeeiro. Em um talhão de 1 ha foram estabelecidos 20 pontos de amostragem em oito ruas de plantas de café e, em cada ponto, foram instalados três conjuntos de armadilhas distantes entre si por um metro, que permaneceram ativas em campo por $48 \mathrm{~h} / \mathrm{semana}$.

A varredura das plantas de café foi realizada com rede entomológica circular, de $35 \mathrm{~cm}$ de diâmetro, de forma aleatória, preferencialmente no terço inferior das plantas. Foram realizados oito ciclos de varreduras em ruas de plantas de aproximadamente, $100 \mathrm{~m}$ de comprimento.

O material obtido foi transferido para frascos plásticos devidamente etiquetados contendo álcool etílico a $70 \%$ e encaminhados ao Laboratório de Sistemática e Bioecologia de Parasitóides e Predadores da Apta Regional Centro Leste (LSBPP) para a triagem e identificação dos hemerobiídeos. A identificação dos hemerobiídeos capturados foi baseada nos artigos de González Olazo (1981), Penny \& Monserrat (1983), Monserrat (1996, 1997, 2000) e Oswald (1988, 1990, 1993).

Análises Faunística e Estatística. Para a análise de diversidade da fauna de hemerobiídeos foram aplicados os índices de Shannon-Wiener (H') e de equitabilidade J (ShannonWiener); para as estimativas dos valores destes índices optouse pelo logaritmo de base 10 e os cálculos foram obtidos com o software DivEs (Rodrigues 2005); os valores de diversidade encontrados foram comparados pelo teste " $\mathrm{t}$ " descrito por Magurran (1988). A constância foi calculada segundo Silveira Neto et al. (1976) e as espécies classificadas como constantes (C $>50 \%)$, acessórias $(25 \%<\mathrm{C}<50 \%)$ e acidentais $(\mathrm{C}<25 \%)$ segundo Bodenheimer (1955), apud Silveira Neto op. cit.

O índice de correlação de Spearman (r), obtido com o auxílio do software SAS/System (2003), foi utilizado para estabelecer a possível relação entre a abundância semanal dos hemerobiídeos, a precipitação pluviométrica e as temperaturas máxima e mínima observadas na semana anterior às coletas. Esse índice foi aplicado tanto para a fauna total de hemerobiídeos quanto para os gêneros coletados. Os dados meteorológicos foram cedidos pelo Escritório de Desenvolvimento Rural de Ribeirão Preto (CATI), órgão da 
Secretaria de Agricultura e Abastecimento do Estado de São Paulo. A eficiência dos métodos de amostragem, avaliada através de contagem dos hemerobiídeos coletados entre agosto de 2005 e abril de 2006, foi analisada pelo modelo logístico em parcelas subdivididas no tempo com a ferramenta PROC GENMOD (SAS/System 2003); a distribuição de Poisson - que é um caso particular de Modelos Lineares Generalizados (McCullagh \& Nelder 1989) - é a distribuição padrão usada para dados de contagem.

\section{RESULTADOS EDISCUSSÃO}

Foram coletados 489 exemplares de Hemerobiidae pertencentes a quatro gêneros (Tab. I): Nusalala (231 espécimes $/ 47,2 \%$ do total de hemerobiídeos coletados), Megalomus (110/22,5\%), Hemerobius (104/21,3\%) e Sympherobius (44 / 9\%).

Os quatro gêneros de hemerobiídeos foram coletados através dos três métodos de coleta utilizados: a rede de varredura capturou o maior número de espécimes (254 espécimes / 51,9\% do total de espécimes coletados) seguida pelas armadilhas de Möericke (169 / 34,6\%) e luminosa (66 / $13,5 \%$ ) (Tab. I). A Tabela II apresenta a média do número total de hemerobiídeos coletados por método de amostragem e o contraste entre estes métodos no período de agosto de 2005 a abril de 2006, quando foram utilizados os três métodos de coleta de forma concomitante. A maior média de hemerobiídeos coletados foi obtida com a rede de varredura $(\mathrm{m}=1,8320)$ que diferiu significativamente das armadilhas de Möericke $(\mathrm{m}=$ $1,2219)$ e da luminosa $(m=0,3551)$; entre a armadilha luminosa e a armadilha de Möericke não foi encontrada diferença significativa. Os dados obtidos demonstram que a rede de varredura é a mais indicada para a captura de Hemerobiidae.
O fato de a armadilha luminosa não apresentar diferença significativa da armadilha de Möericke pode estar relacionado com os valores da média de Hemerobiidae capturados com este tipo de armadilha e do seu erro padrão $(m=0,3551 \pm 0,2856)$; o alto valor do erro padrão indica a menor precisão da estimativa desta média, que pode ter ocorrido em função de a armadilha luminosa ter sido operada em apenas dois pontos de amostragem em contrapartida com os 20 pontos para as armadilhas de Möericke e oito pontos para a rede de varredura.

Diversidade dos Hemerobiidae. A armadilha de Möericke foi o método de amostragem que apresentou os maiores valores de diversidade $\left(\mathrm{H}^{\prime}=0,56\right)$ e de equitabilidade $(\mathrm{J}=0,93)$ e capturou exemplares pertencentes aos quatro gêneros. Os valores de diversidade e de equitabilidade para a rede de varredura, armadilha luminosa e o conjunto dos métodos de amostragem foram próximos e inferiores $\left(\mathrm{H}^{\prime}=0,49-0,55\right.$ e $\mathrm{J}=$ 0,81-0,91) aos obtidos com a armadilha de Möericke (Tab. III). O teste " $\mathrm{t}$ " proposto por Magurran (1988) para comparação entre dois valores estimados do índice de diversidade de Shannon mostrou que as diferenças entre os métodos de amostragem utilizados não foram significativas (Tab. III).

$\mathrm{O}$ fato de a rede de varredura ter apresentado valores de diversidade e equitabilidade inferiores aos demais métodos avaliados, porém não significativo, pode estar relacionado com a abundância de exemplares de Nusalala capturados com este aparato de coleta $(53,5 \%$ do total de hemerobiídeos coletados).

Os valores de diversidade obtidos neste estudo são semelhantes aos encontrados por Szentkirályi (1989) em diferentes sistemas de cultivos de milho, monitorado durante seis anos com diferentes métodos de amostragem; a área de cultivo em rotação de culturas com diversidade de vegetação adjacente quando comparadas com monocultura e

Tabela I. Abundância mensal de hemerobiídeos coletados com armadilhas de Möericke, luminosa e rede de varredura, em Coffea arabica L., no período de 2005/2006, em Cravinhos (SP).

\begin{tabular}{|c|c|c|c|c|c|c|c|c|c|c|c|c|c|c|c|c|c|c|c|c|}
\hline \multirow[b]{2}{*}{$\begin{array}{c}\text { mês } \\
\text { de } \\
\text { coleta }\end{array}$} & \multicolumn{5}{|c|}{ Armadilha de Möericke } & \multicolumn{5}{|c|}{ Armadilha Luminosa } & \multicolumn{5}{|c|}{ Rede de varredura } & \multicolumn{5}{|c|}{$\sum$ amostragens } \\
\hline & 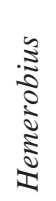 & 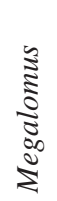 & $\begin{array}{l}\frac{z}{3} \\
\frac{3}{3} \\
\vdots\end{array}$ & 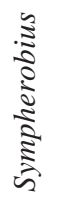 & 芜 & 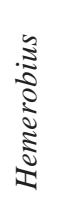 & 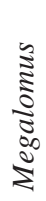 & $\begin{array}{c}\frac{z}{3} \\
\frac{\pi}{3} \\
\frac{3}{3}\end{array}$ & 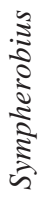 & స్త్ర & 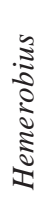 & 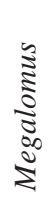 & $\frac{\frac{\tilde{D}}{\tilde{J}}}{\frac{\tilde{S}}{3}}$ & 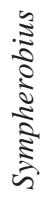 & 胥 & 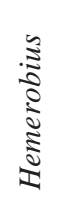 & 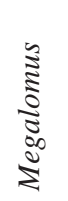 & 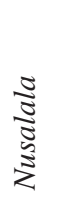 & 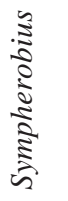 & 胥 \\
\hline mai./05 & 0 & 0 & 0 & 0 & 0 & 0 & 0 & 0 & 0 & 0 & - & - & - & - & - & 0 & 0 & 0 & 0 & 0 \\
\hline jun./05 & 7 & 2 & 4 & 0 & 13 & 6 & 0 & 1 & 0 & 7 & - & - & - & - & - & 13 & 2 & 5 & 0 & 20 \\
\hline jul./05 & 9 & 4 & 5 & 0 & 18 & 1 & 0 & 0 & 0 & 1 & - & - & - & - & - & 10 & 4 & 5 & 0 & 19 \\
\hline ago./05 & 5 & 2 & 5 & 1 & 13 & 12 & 0 & 11 & 1 & 24 & 6 & 4 & 4 & 0 & 14 & 23 & 6 & 20 & 2 & 51 \\
\hline set./05 & 18 & 4 & 9 & 1 & 32 & 0 & 1 & 0 & 0 & 1 & 5 & 3 & 6 & 0 & 14 & 23 & 8 & 15 & 1 & 47 \\
\hline out./05 & 3 & 1 & 2 & 1 & 7 & 1 & 0 & 1 & 1 & 3 & 5 & 3 & 3 & 4 & 15 & 9 & 4 & 6 & 6 & 25 \\
\hline nov./05 & 1 & 1 & 9 & 8 & 19 & 0 & 0 & 4 & 0 & 4 & 6 & 4 & 14 & 7 & 31 & 7 & 5 & 27 & 15 & 54 \\
\hline dez./05 & 5 & 3 & 6 & 9 & 23 & 0 & 2 & 2 & 1 & 5 & 4 & 9 & 15 & 2 & 30 & 9 & 14 & 23 & 12 & 58 \\
\hline jan./06 & 1 & 5 & 14 & 2 & 22 & 3 & 5 & 5 & 1 & 14 & 3 & 10 & 29 & 1 & 43 & 7 & 20 & 48 & 4 & 79 \\
\hline fev./06 & 1 & 3 & 6 & 1 & 11 & 0 & 5 & 0 & 1 & 6 & 2 & 12 & 20 & 2 & 36 & 3 & 20 & 26 & 4 & 53 \\
\hline mar./06 & 0 & 0 & 9 & 0 & 9 & 0 & 0 & 0 & 0 & 0 & 0 & 17 & 38 & 0 & 55 & 0 & 17 & 47 & 0 & 64 \\
\hline abr./06 & 0 & 0 & 2 & 0 & 2 & 0 & 1 & 0 & 0 & 1 & 0 & 9 & 7 & 0 & 16 & 0 & 10 & 9 & 0 & 19 \\
\hline total & 50 & 25 & 71 & 23 & 169 & 23 & 14 & 24 & 5 & 66 & 31 & 71 & 136 & 16 & 254 & 104 & 110 & 231 & 44 & 489 \\
\hline
\end{tabular}

- coletas não realizadas 
Tabela II. Número médio de hemerobiídeos coletados por método de amostragem e contraste das médias, através do Proc Genmod, em cultivo de café, no período de agosto de 2005 a abril de 2006, em Cravinhos (SP).

\begin{tabular}{lllll}
\hline \multirow{2}{*}{$\begin{array}{l}\text { métodos de } \\
\text { amostragem }\end{array}$} & \multicolumn{4}{c}{ total de Hemerobiidae } \\
\cline { 2 - 5 } & média $\pm \mathrm{EP}$ & $\mathrm{X}^{2}$ & $\mathrm{p}$ & resultado \\
\hline $\begin{array}{l}\text { armadilha luminosa } \\
(\mathrm{AL})\end{array}$ & $0,3551 \pm 0,2856$ & & \\
$\begin{array}{l}\text { armadilha de Möericke } \\
(\mathrm{AM})\end{array}$ & $1,2219 \pm 0,1148$ & & \\
$\begin{array}{l}\text { rede de varredura (RV) } \\
\text { contraste entre AM e }\end{array}$ & $1,8320 \pm 0,0904$ & & \\
$\begin{array}{l}\text { AL } \\
\text { contraste entre AM e }\end{array}$ & 2,56 & 0,1097 & $\mathrm{AM}=\mathrm{AL}$ \\
$\mathrm{RV}$ & 4,03 & 0,0448 & $\mathrm{AM} \neq \mathrm{RV}$ \\
contraste entre AL e & 3,60 & 0,0577 & $\mathrm{AL} \neq \mathrm{RV}$ \\
$\mathrm{RV}$ & & & & \\
\hline
\end{tabular}

$\mathrm{EP}=$ erro padrão da média

$\mathrm{p}=$ nível de significância

monocultura com vegetação adjacente apresentou maior valor de riqueza de espécies $(S=10)$ e de diversidade de espécies (índice de Shannon-Wiener=0,49).

Todos os gêneros de hemerobiídeos coletados foram constantes no cafeeiro (Tab. IV): Hemerobius $(\mathrm{C}=69,2 \%)$, Megalomus $(\mathrm{C}=84,6 \%)$, Nusalala $(\mathrm{C}=92,3 \%)$ e Sympherobius $(\mathrm{C}=53,8 \%)$. A constância de Nusalala foi registrada em todo o período de amostragem, à exceção do mês de maio de 2005, quando foi realizada uma aplicação de defensivo químico (thiamethoxam) para o controle de L. coffeella. Hemerobius foi constante de junho de 2005 a fevereiro de 2006, Megalomus o foi de junho de 2005 a abril de 2006 e Sympherobius de agosto de 2005 a fevereiro de 2006.

Os valores de constância encontrados para os gêneros Megalomus e Nusalala indicam que, após a sua identificação específica, a atividade predatória daquelas espécies deve ser pesquisada para uma possível utilização em programas de controle biológico de pragas.

Atividade sazonal dos Hemerobiidae. Adistribuição sazonal dos adultos de hemerobiídeos pode ser observada na Figura 1. A população de hemerobiídeos esteve presente na área estudada durante o ano todo. A não captura de exemplares de hemerobiídeos em maio de 2005 pode ser creditada a aplicação de defensivo químico (thiamethoxam) para o controle de bicho-
Tabela III. Índices de Diversidade de Shannon-Wiener (H') e de Equitabilidade (J) da população de Hemerobiidae coletada com armadilhas de Möericke, luminosa e da rede de varredura e comparação, através do teste $\mathrm{t}$, dos valores de diversidade (H'), em Coffea arabica L. cv. Obatã, no período de maio de 2005 a abril de 2006, em Cravinhos (SP).

\begin{tabular}{|c|c|c|c|}
\hline \multirow[b]{2}{*}{ métodos de amostragem } & \multicolumn{3}{|c|}{ total de Hemerobiidae } \\
\hline & $\mathrm{H}^{\prime}$ & $\mathrm{J}$ & $\mathrm{t}_{\text {calculado }}$ \\
\hline rede de varredura (RV) & 0,49 & 0,81 & \\
\hline armadilha luminosa (AL) & 0,55 & 0,91 & \\
\hline armadilha de Moericke (AM) & 0,56 & 0,93 & \\
\hline$\sum$ métodos de coleta $(\Sigma \mathrm{M})$ & 0,54 & 0,89 & \\
\hline comparação entre $\mathrm{H}_{\mathrm{RV}}^{\prime}$ e $\mathrm{H}_{\mathrm{AL}}^{\prime}$ & & & $-0,003^{\mathrm{ns}}$ \\
\hline comparação entre $\mathrm{H}_{\mathrm{RV}}{ }_{\mathrm{RV}}$ e $\mathrm{H}_{\mathrm{AM}}{ }_{\mathrm{AL}}$ & & & $-0,004^{\mathrm{ns}}$ \\
\hline comparação entre $\mathrm{H}^{\prime}{ }_{\mathrm{RV}} \mathrm{e} \mathrm{H}^{\prime}{ }^{\prime}{ }_{M}$ & & & $-0,002^{\mathrm{ns}}$ \\
\hline comparação entre $\mathrm{H}^{{ }_{\mathrm{AL}}}{ }_{\mathrm{AL}}$ e $\mathrm{H}_{\mathrm{AM}}$ & & & $-0,001^{\mathrm{ns}}$ \\
\hline comparação entre $\mathrm{H}_{\mathrm{AL}}{ }_{\mathrm{AL}}$ e $\mathrm{H}^{\prime}{ }_{\mathrm{MM}}$ & & & $0,00009^{\text {ns }}$ \\
\hline comparação entre $\mathrm{H}_{\mathrm{AM}}{ }^{\mathrm{AL}} \mathrm{e}^{\prime}{ }^{\prime}{ }_{\mathrm{M}}$ & & & $0,0008^{\mathrm{ns}}$ \\
\hline
\end{tabular}

${ }^{\mathrm{ns}}=$ não significativo

mineiro L. coffeella. As maiores freqüências foram registradas em agosto e setembro (antes e durante a floração das plantas de café), na primavera e durante o verão (quando, em seu início, os cafeeiros apresentavam grãos "chumbinho"). O maior pico populacional foi observado na metade do verão, em janeiro. A menor frequiência de hemerobiídeos observada no mês de outubro, quando comparada aos demais meses da primavera, pode também estar relacionada à aplicação de defensivo químico (ditiocarbamato) para o controle de bicho-mineiro.

Os gêneros Nusalala e Megalomus apresentaram maiores frequiências na primavera e no verão, Hemerobius no final do inverno e início da primavera e Sympherobius no final da primavera (Fig. 2). Para todos os gêneros foram observados dois picos de freqüência bem definidos: Nusalala em janeiro e março, Megalomus em janeiro e fevereiro, Hemerobius em agosto e setembro e Sympherobius em novembro e dezembro, o que sugere um padrão temporal de uso do ambiente por estes predadores.

$\mathrm{Na}$ Hungria e na França, nos meses de julho e agosto (verão), ocorrem os picos de vôos das espécies de hemerobiídeos mais freqüentes, principalmente de espécies pertencentes aos gêneros Hemerobius, Micromus, Psectra, Sympherobius e Wesmaellus (Szabó \& Szentkirályi 1981; Szentkirályi 1992; Trouvé et al. 2002).

Tabela IV. Constância de hemerobiídeos associados a Coffea arabica L., no período de maio de 2005 a abril de 2006, em Cravinhos (SP).

\begin{tabular}{|c|c|c|c|c|c|c|c|c|c|c|c|c|c|c|}
\hline Hemerobiidae & mai. & jun. & jul. & ago. & set. & out. & nov. & dez. & jan. & fev. & mar. & abr. & total & Constância \\
\hline Hemerobius & 0 & 13 & 10 & 23 & 23 & 9 & 7 & 9 & 7 & 3 & 0 & 0 & 104 & constante \\
\hline Megalomus & 0 & 2 & 4 & 6 & 8 & 4 & 5 & 14 & 20 & 20 & 17 & 10 & 110 & constante \\
\hline Nusalala & 0 & 5 & 5 & 20 & 15 & 6 & 27 & 23 & 48 & 26 & 47 & 9 & 231 & constante \\
\hline Sympherobius & 0 & 0 & 0 & 2 & 1 & 6 & 15 & 12 & 4 & 4 & 0 & 0 & 44 & constante \\
\hline Abundância & 0 & 20 & 19 & 51 & 47 & 25 & 54 & 58 & 79 & 53 & 64 & 19 & 489 & \\
\hline $\mathrm{n}^{\mathrm{o}}$ de gêneros & 0 & 3 & 3 & 4 & 4 & 4 & 4 & 4 & 4 & 4 & 2 & 2 & 4 & \\
\hline
\end{tabular}




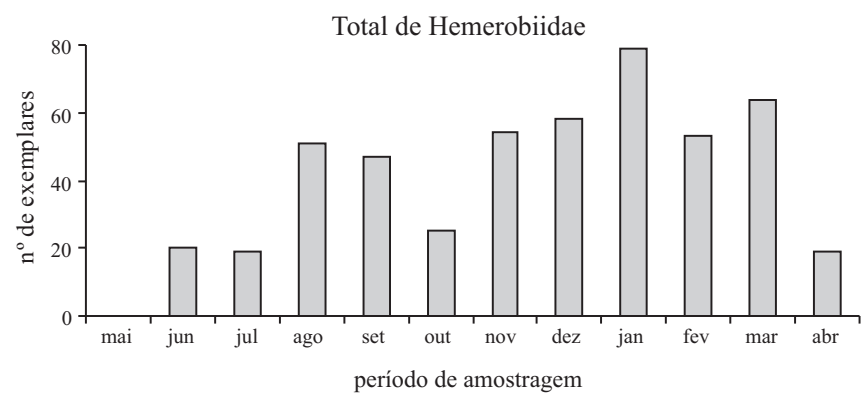

Fig. 1. Número total de Hemerobiidade coletados mensalmente, em cultivo de café no período de maio de 2005 a abril de 2006, em Cravinhos $(\mathrm{SP})$.

Hemerobiidae x precipitação pluviométrica e temperatura. Nas Figuras 3 e 4 são apresentados os dados referentes às precipitações pluviométricas acumuladas e as médias de temperaturas máximas e mínimas durante as estações do ano no decorrer do estudo. A precipitação pluviométrica total, entre maio de 2005 e abril de 2006 foi de $1.340,6$ mm; agosto foi o mês mais seco com ausência de precipitação pluviométrica e fevereiro o mais chuvoso, com 297,1 mm precipitação pluviométrica (Fig. 3).

No período de maio de 2005 a abril de 2006, os maiores valores de temperaturas máximas ocorreram a partir de outubro e, durante o verão, oscilaram entre $29,2-37,1^{\circ} \mathrm{C}$; os menores valores de temperaturas mínimas ocorreram no inverno e oscilaram entre $9,6-16,9^{\circ} \mathrm{C}$ (Fig. 4).

Quanto à precipitação pluviométrica, foi verificada correlação positiva e significativa $(\mathrm{p}<0,05)$ com a abundância de Megalomus (r=0,44), não houve correlação significativa com Hemerobius, Nusalala, Sympherobius e com o total de
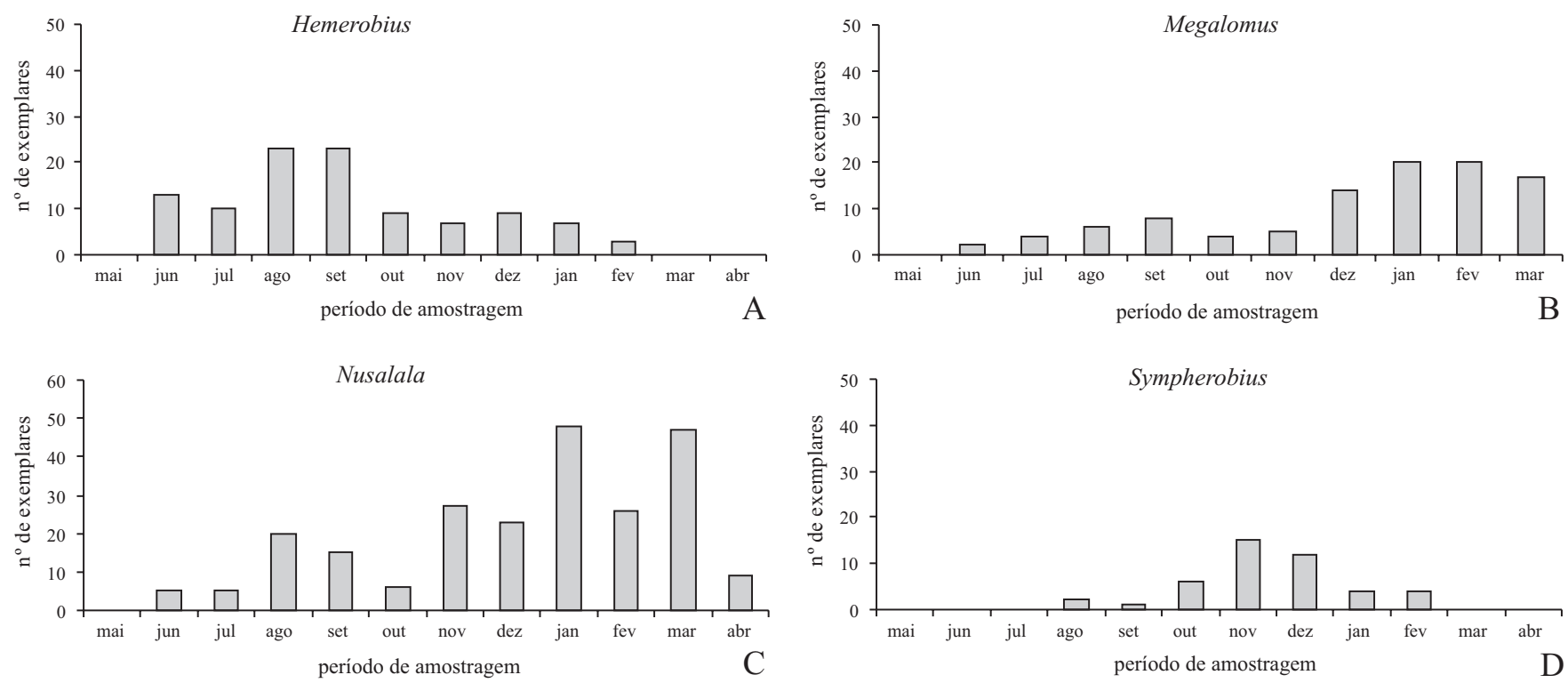

Fig. 2. Número total de Hemerobiidade coletados por gênero, mensalmente, em cultivo de café no período de maio de 2005 a abril de 2006 , em Cravinhos (SP).
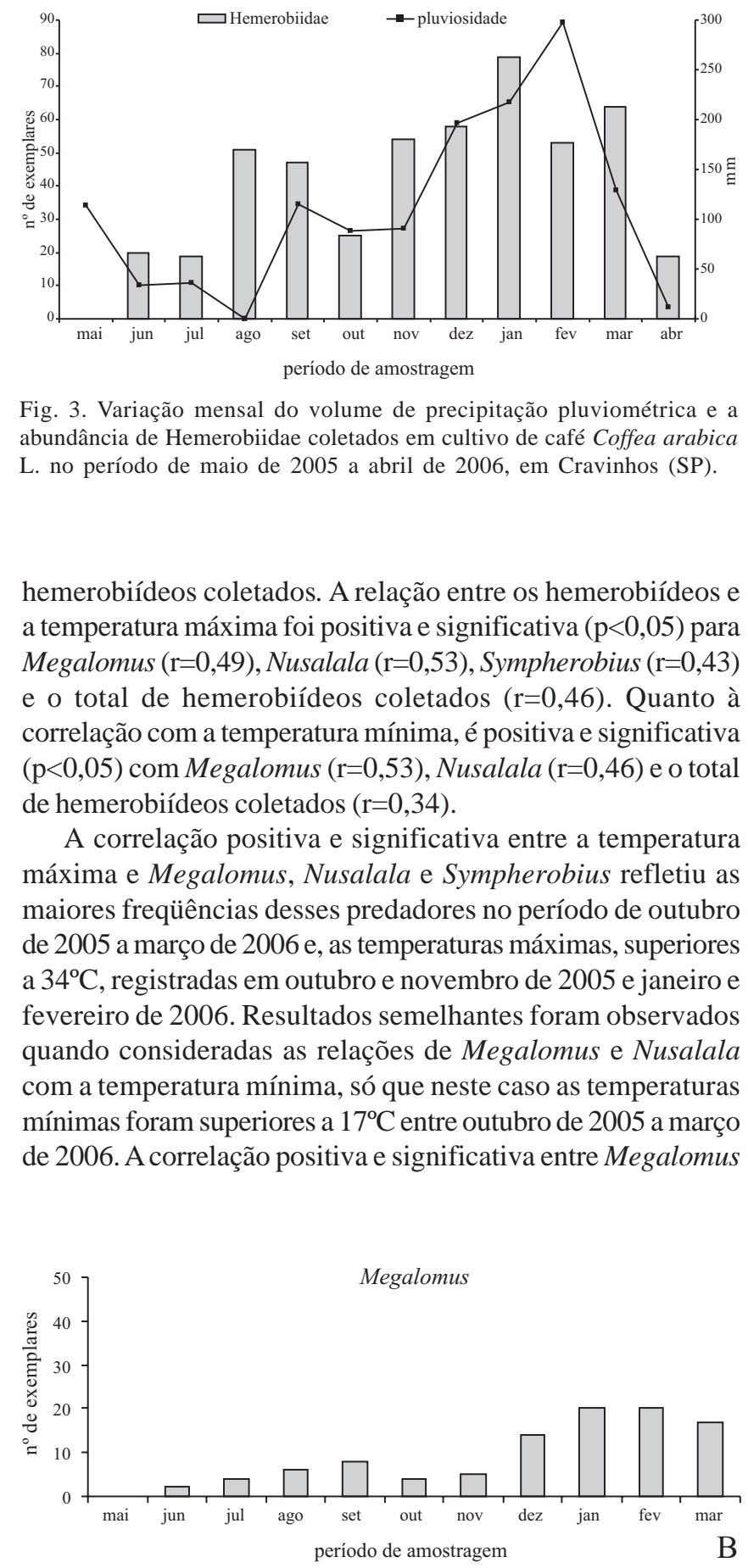

Fig. 3. Variação mensal do volume de precipitação pluviométrica e a abundância de Hemerobiidae coletados em cultivo de café Coffea arabica L. no período de maio de 2005 a abril de 2006, em Cravinhos (SP).

hemerobiídeos coletados. A relação entre os hemerobiídeos e a temperatura máxima foi positiva e significativa $(\mathrm{p}<0,05)$ para Megalomus $(\mathrm{r}=0,49)$, Nusalala $(\mathrm{r}=0,53)$, Sympherobius $(\mathrm{r}=0,43)$ e o total de hemerobiídeos coletados $(\mathrm{r}=0,46)$. Quanto à correlação com a temperatura mínima, é positiva e significativa $(\mathrm{p}<0,05)$ com Megalomus $(\mathrm{r}=0,53)$, Nusalala $(\mathrm{r}=0,46)$ e o total de hemerobiídeos coletados $(\mathrm{r}=0,34)$.

A correlação positiva e significativa entre a temperatura máxima e Megalomus, Nusalala e Sympherobius refletiu as maiores freqüências desses predadores no período de outubro de 2005 a março de 2006 e, as temperaturas máximas, superiores a $34^{\circ} \mathrm{C}$, registradas em outubro e novembro de 2005 e janeiro e fevereiro de 2006. Resultados semelhantes foram observados quando consideradas as relações de Megalomus e Nusalala com a temperatura mínima, só que neste caso as temperaturas mínimas foram superiores a $17^{\circ} \mathrm{C}$ entre outubro de 2005 a março de 2006. A correlação positiva e significativa entre Megalomus 


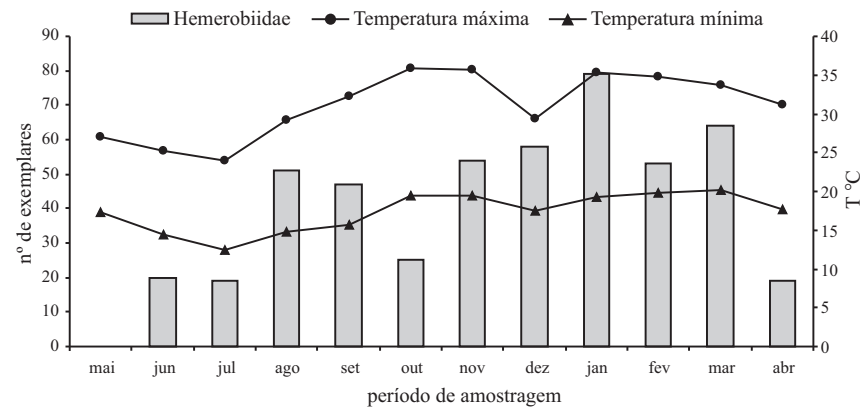

Fig. 4. Variação mensal da temperatura máxima e mínima e a abundância de Hemerobiidae coletados em cultivo de café Coffea arabica L. no período de maio de 2005 a abril de 2006, em Cravinhos (SP).

e a precipitação pluviométrica refletiu a maior abundância desse gênero em janeiro e fevereiro, meses em que foram registrados os maiores valores de pluviosidade.

Os valores de " $r$ " revelaram correlações fracas e moderadas entre os gêneros de hemerobiídeos, a chuva e as temperaturas máxima e mínima e sugerem que estes não foram os únicos fatores determinantes da flutuação populacional desses predadores e evidencia que a sua abundância também está relacionada com a disponibilidade de presas.

Agradecimento. Ao Sr. Edson Minohara, proprietário da Fazenda Palmares, pela cessão da área para realização deste estudo.

\section{REFERÊNCIAS}

Ábrahám, L.; V. Markó \& J. Vas. 2003. Investigations on a Neuropteroid community by using different methods. Acta Phytopathologica et Entomologica Hungarica 38: 199-207.

Almeida, L. M.; C. S. Ribeiro-Costa \& L. Marinoni. 1998. Manual de coleta, conservação, montagem e identificação de insetos. Ribeirão Preto, Holos, 78 p.

Carpenter, F. M. 1940. A revision of the Neartic Hemerobiidae, Berothidae, Sisyridae, Polystoechotidae and Dilaridae (Neuroptera). Proceedings of the American Academy of Arts and Sciences 74: 193-278.

Chagas, E. F. das; S. Silveira Neto; A. J. B. P. Braz; C. P. B. Mateus \& I. P. Coelho. 1982. Flutuação populacional de pragas e predadores em citros. Pesquisa Agropecuária Brasileira 17: 817-824.

Ecole, C. C.; R. A. Silva; J. N. C. Louzada; J. C. Moraes; L. R. Barbosa \& B. G. Ambrogi. 2002. Predação de ovos, larvas e pupas do bichomineiro-do-cafeeiro, Leucoptera coffeella (Guérin-Menèville \& Perrottet, 1842) (Lepidoptera: Lyonetiidae) por Chrysoperla externa (Hagen, 1861) (Neuroptera: Chrysopidae). Ciências e Agrotecnologia 26: 318-324.

Fragoso, D. B.; P. Jusselino-Filho; R. N. C. Guedes \& R. Proque. 2001. Seletividade de inseticidas a vespas predadoras de Leucoptera coffeella (Guér.-Menèv.) (Lepidoptera: Lyonetiidae). Neotropical Entomology 30: 139-143.

González Olazo, E. V. 1981. El genero Megalomus Rambur (Neurop. Planipennia - Hemerobiidae) en Argentina y Chile. Acta Zoológica Lilloana 36: 97-113.

Gravena, S. 1983. Táticas de manejo integrado do bicho mineiro do cafeeiro Perileucoptera coffeella (Guérin-Menèville, 1842): II Amostragem da praga e de seus inimigos naturais. Anais da Sociedade Entomológica do Brasil 12: 273-281.

Gravena, S. 1992. Manejo ecológico de pragas do cafeeiro.
Jaboticabal: Funep, 30 p. (Boletim Técnico, 3).

Lara, R. I. R. \& S. de Freitas. 2002. Caracterização morfológica de adultos de Nusalala tessellata (Gerstaecker, 1888) (Neuroptera, Hemerobiidae). Revista Brasileira de Entomologia 46: $523-$ 530.

Lara, R. I. R. \& S. de Freitas. 2003. Caracterização morfológica de espécies de Hemerobius Linnaeus, 1758 (Neuroptera, Hemerobiidae) associadas a cultivo de café (Coffea arabica L.), milho (Zea mays L.) e erva-mate (Ilex paraguariensis St. Hill.). Revista Brasileira de Entomologia 47: 427-434.

Lara, R. I. R. \& N. W. Perioto. 2003. Primeiro registro de ocorrência de Sympherobius miranda (Navás, 1920) (Neuroptera, Hemerobiidae) para o Brasil. Arquivos do Instituto Biológico 70: $511-512$.

Magurran, A. E. 1988. Ecological diversity and its measurements. Princeton, Princeton University Press, 179 p.

McCullagh, P. \& J. A. Nelder. 1989. Generalized Linear Models. London: Chapman \& Hall, 511 p.

Monserrat, V. J. 1990. A systematic checklist of the Hemerobiidae of the world (Insecta: Neuroptera). In: INTERNATIONAL SYMPOSIUM ON NEUROPTEROLOGY, 3, Pretoria. Advances in Neuropterology; Proceedings...Pretoria, p. 215-262.

Monserrat, V. J. 1996. Revisión del género Hemerobius de Latinoamerica (Neuroptera, Hemerobiidae). Fragmenta Entomologica 27: 399-523.

Monserrat, V. J. 1997. Revisión del género Megalomus de Latinoamérica (Neuroptera, Hemerobiidae). Fragmenta Entomologica 29: 123-206.

Monserrat, V. J. 2000. Revisión del género Nusalala (Neuroptera, Hemerobiidae). Fragmenta Entomologica 32: 83-162.

Oswald, J. D. 1988. A revision of the genus Sympherobius Banks (Neuroptera, Hemerobiidae) of America North of Mexico with a synonymical list of the world species. Journal of the New York Entomological Society 96: 390-451.

Oswald, J. D. 1990. Revision of the Neotropical brown lacewing genus Nomerobius (Neuroptera, Hemerobiidae). Annals of the Entomological Society of America 83: 18-29.

Oswald, J. D. 1993. Revision and cladistic analysis of the world genera of the family Hemerobiidae (Insecta: Neuroptera). Journal of the New York Entomological Society 101: 143-299.

Parra, J. R. P.; W. Gonçalves; S. Gravena \& A. R. Marconato. 1977. Parasitos e predadores do bicho-mineiro do cafeeiro Perileucoptera coffeella (Guérin-Mèneville, 1842) em São Paulo. Anais da Sociedade Entomológica do Brasil 6: 138-143.

Penny, N. D. \& V. J. Monserrat. 1983. Neuroptera of the Amazon basin Part 10-Hemerobiidae. Acta Amazônica 13: 879-909.

Perioto, N. W.; R. I. R. Lara; J. C. C. Santos \& T. C. da Silva. 2000. Utilização de armadilhas de Möericke em ensaios de seletividade em himenópteros parasitóides. Arquivos do Instituto Biológico 67: 93.

Reis, P. R.; J. C. de Souza \& M. Venzon. 2002. Manejo Ecológico das principais pragas do cafeeiro. Informe Agropecuário 23: 83-99.

Rodrigues, W. C. 2005. DivEs - Diversidade de espécies. Versão 2.0. Software e Guia do Usuário. Disponível em: <http:// www.ebras.vbweb.com.br> Acesso em 10 maio 2005.

SAS/STAT. 2003. User's guide: statistics, version 9, v.1, SAS Institute Inc., Cary, NC, USA.

Silveira Neto, S.; O. Nakano; D. Barbin \& N. A. Villa Nova. 1976. Manual de ecologia dos insetos. São Paulo: Agronômica Ceres, $419 \mathrm{p}$.

Souza, B. 1999. Aspectos morfológicos de adultos de Nusalala uruguaya (Navás, 1923) (Neuroptera: Hemerobiidae). Ciências e Agrotecnologia 23: 252-257.

Souza, B. \& A. I. Ciociola. 1995. Aspectos comportamentais de Nusalala uruguaya (Navás) (Neuroptera: Hemerobiidae), em laboratório. Anais da Sociedade Entomológica do Brasil, 24: 173-175.

Souza, B. \& A. I. Ciociola. 1997. Efeito de diferentes dietas sobre a fecundidade e longevidade de Nusalala uruguaya (Navás, 1923) (Neuroptera: Hemerobiidae). Pesquisa Agropecuária Brasileira 32: $27-32$. 
Souza, B.; A. I. Ciociola \& J. C. Matioli. 1989. Biologia comparada de Nusalala uruguaya (Navás, 1923) (Neuroptera: Hemerobiidae) alimentada com diferentes espécies de afídeos. II. Fase de prépupa, pupa e adulta. Anais da Sociedade Entomológica do Brasil 18: $43-51$

Souza, B.; J. C. Matioli \& A. I. Ciociola. 1990. Biologia comparada de Nusalala uruguaya (Navás, 1923) (Neuroptera: Hemerobiidae) alimentada com diferentes espécies de afídeos. I. fase de larva. Anais da Escola Superior de Agricultura "Luiz de Queiroz" 47: 283-300.

Szabó, S. \& F. Szentkirályi. 1981. Communities of Chrysopidae and Hemerobiidae (Neuroptera) in some apple-orchards. Acta Phytopathologica Academiae Scientiarum Hungaricae 16: 157-169.

Szentkirályi, F. 1989. Aphidophagous Crysopid and Hemerobiid (Neuropteroidea) subguilds in different maize fields: influence of vegetational diversity on sub guild structure. Acta
Phytopathologica et Entomologica Hungarica 24: 207-211. Szentkirályi, F. 1992. Spatio-temporal patterns of brown lacewings based on the Hungarian light trap network (Insecta: Neuroptera: Hemerobiidae). In: INTERNATIONAL SYMPOSIUM ON NEUROPTEROLOGY, 4, Toulouse. Advances in Neuropterology; Proceedings... Toulouse. p. 349-357.

Szentkirályi, F. 1997. Seasonal flight patterns of some common brown lacewing species (Neuroptera, Hemerobiidae) in Hungarian agricultural regions. Biologia 52: 291-302.

Szentkirályi, F. 2002. Fifty-year-long insect survey in Hungary: T. Jermy's contributions to light-trapping. Acta Zoologica Academiae Scientiarum Hungaricae 48: 85-105.

Trouvé, C.; D. Thierry \& M. Canard. 2002. Preliminary survey of the lacewings (Neuroptera, Chrysopidae, Hemerobiidae) in agroecosytems in Northern France, with phonological notes. Acta Zoologica Academiae Scientiarum Hungaricae 48: 359-369. 